\title{
Power Electronic Courses That Work
}

\author{
Taufik \\ Electrical Engineering Department, California Polytechnic State University \\ San Luis Obispo, CA 93407 taufik@calpoly.edu
}

\begin{abstract}
This paper presents approaches that have been implemented in power electronic courses to be more appealing to electrical engineering students and hence increase their enrollments. The approaches entail redesigning the structure and content of the courses along with developing new laboratory experiments to better align the lab with the lecture portion of the courses and to reflect recent practical issues in power electronics. In addition to lab experiments, the redesigned courses provide students with an opportunity to apply their knowledge in assigned hardware projects that illustrated many of the points learned in the lecture portion of the course with increasing level of difficulties. Impact on student enrollments in these redesigned courses will be discussed.
\end{abstract}

Index Terms - Electrical Engineering courses, Engineering Lab, Power Electronics.

\section{INTRODUCTION}

Despite concern for the declining interest in science and engineering among U.S. students, data from the National Science Foundation (NSF) has actually shown that overall the fraction of U.S. undergraduates choosing to major in science and engineering has stayed remarkably constant, roughly one in three, for more than a generation [1]. The data further show that there have been shifts within the natural sciences and engineering, as interest in particular fields have increased and decreased. One such field that has recently been struggling with decreasing level of interest is one branch of electrical engineering known as power engineering. Low enrollment in the field causes the difficulty faced by utility companies in hiring graduating seniors in power [2]. One power course that is typically offered in many universities, often as technical elective course, is power electronics.

Power Electronics courses at Cal Poly are some of the many technical elective courses offered to Electrical Engineering students. Currently, three power electronic courses are available as a sequence at Cal Poly and each is offered once a year. Traditionally only students focusing in power take these courses. However, due to increased widespread applications of power electronics, the courses naturally should also be suitable as technical electives for the non-power electrical engineering majors. Prior to Fall 1999, the contents of power electronic courses with their associated labs at Cal Poly were geared toward mainly high power applications. This was observed to have caused a prevalent misconception among our electrical engineering students that power electronics were solely for power students. Furthermore, the course contents lacked the focus to recent development and wider applications of power electronic technology. As a result and coupled with the overall low interest in power, low enrollment (as low as 8 students in 1999) in power electronic courses was encountered before the redesigning took place.

This paper explains approaches that were undertaken in reshaping the power electronic courses along with their associated lab experiments. The immediate objectives were to increase student enrollment in these courses and to teach students the broad engineering concepts and techniques in power electronics and provide them with hands-on engineering learning experiences through lab experiments of commonly used power electronic circuits. The long-term objective was to attract more students into power and to further prepare students for design and instrumentation skills that they will likely enter upon graduation.

\section{Historical Perspective}

Cal Poly has long has the reputation to have a strong undergraduate power program in California and has been one of the few undergraduate universities in California that has produced power engineers. However, in 1990's, the number of students in the power program has continuously declined, mainly due to the information technology boom. When joining Cal Poly in 1999, the author was given the task to help rejuvenate the power program in general and to develop the power electronic courses in particular.

Every electrical engineering student normally selects their area of concentration in as early as the third year, shortly after they have taken one required power course called Energy Conversion Electromagnetics. When students choose power, the first technical elective course they usually take is the Power System Analysis I. Beyond this, the students may then choose at least two more technical elective power courses with two labs to fulfill the 12 unit technical elective requirement. The following lists power courses along with their quarter units that are currently available as technical elective:

- $\quad$ EE 406 - Power System Analysis I (4 units)

- $\quad$ EE 407 - Power System Analysis II (4 units)

- $\quad$ EE 518 - Power System Protection (4 units)

- $\quad$ EE 444 - Power Systems Lab (1 unit)

- $\quad$ EE 417 - AC Machines with Lab (4 units)

- $\quad$ EE 410 - Power Electronics I with Lab (4 units) 
- $\quad$ EE 411 - Power Electronics II with Lab (4 units)

- $\quad$ EE 527 - Advanced Power Electronics (4 units)

Within power electronics, as listed in the previous list, there are currently three technical electives: EE 410, EE 411, and EE 527. EE 410 and EE 411 had been continuously offered annually but just like other power courses their enrollment has been declining in 1999. The lab portions of EE 410 and 411 consisted of old lab experiments that needed to be updated. The last course EE 527, prior to 2002, had not been offered for quite a while due to extremely low enrollment (less than 5).

\section{MOTIVATION AND OPPORTUNITY}

Power Electronics is a study of how to process and control the flow of electric energy by supplying voltages and currents in a form that is optimally suited for user loads [3]. Power electronics is an enabling technology which is the key to efficient electrical energy conversion which becomes important in today's energy conservative society. In addition, power electronics offers a very wide range of applications from low to high power. In the low power level, power electronics is used in the battery-powered portable electronics, home audio systems, computers, and in practically any home appliances. In the medium level, power electronics finds its use in applications such as ac motor controls and automotive. Recent emergence of power semiconductor devices has brought power electronics into the high power domain such as those used for converters and controllers in bulk power transmission. Another factor that boosts the popularity of power electronic is the conversion required in renewable energy as a result of global awareness in going away from fossil-based fuel for energy.

Another motivation to rejuvenate the power program comes from the issues related to the aging of active power engineers and expected retirements in near future and the present state of electric grid [4]. Utilities and the industry face new challenges to exploit new technologies including power electronics, distributed generation and new materials. All of these should pose great motivations for students to go into this new era of power engineering, as well as an opportunity for the author to redesign power electronic courses and labs to be more appealing to students.

\section{Redesigning The LeCture Courses}

In order to attract more than just power students into taking power electronic courses, the following approaches were implemented when redesigning the courses:

- First course (EE 410) should cover ALL basic conversion concepts (ac to dc, dc to dc, dc to ac and ac to ac)

- First course (EE 410) should include practical examples covering mostly low power applications
- First course (EE 410) should include a hardware project assignment in basic low-power dc-dc converter commonly used in high-level system design

- Second course (EE 411) should cover recent issues in power electronics such as synchronous rectifier, softswitching, dc transmission, FACTS controllers, power quality and ac drives

- Second course (EE 411) should provide examples of applications in a mixed power level: low, medium and high power

- Second course (EE 411) should include a hardware project assignment in low-power dc-dc converter commonly used in low-level system design

- Third course (EE 527) should focus more into any one of the advanced topics and recent development in power electronics

- Third course (EE 527) should include a hardware project assignment with a higher level of complexity such as the isolated dc-dc topology

In the first course, the content was changed to cover all basic conversion concepts with focus on functionalities of each conversion and applications in low power. The later is particularly challenging since traditionally power electronic is commonly used for tens of watts to tens of kilowatts applications. However, nowadays power electronics finds its applications in very low power, as low as milli-watt applications such as those found in cell-phones. This approach is much needed for the course to be more appealing to nonpower students. Below is the list of topics covered in EE 410:

- Power Electronics: What, Where and Why

- $\mathrm{AC}$ to fixed DC conversion

- $\mathrm{AC}$ to adjustable DC conversion

- $\mathrm{AC}$ to $\mathrm{AC}$ conversion

- $\mathrm{DC}$ to DC non-isolated conversion

- $\mathrm{DC}$ to DC isolated conversion

- $\mathrm{DC}$ to $\mathrm{AC}$ conversion

Another change in the course is the method in which the course materials are covered. The method is to first discuss each conversion from the big picture (system) and then analyze the system by breaking it down into smaller pieces. For example, when discussing dc-dc converter, the overall objective, functionalities of each element and characteristic of ideal converter are first presented. Then, for each different dcdc topology, their unique properties and characteristics are discussed and compared to one another and with the ideal one. This approach enables the students to understand the whole picture of the conversion down to sufficient details and makes the course materials easier to understand by the electrical engineering students and people without electrical engineering background.

To expose students to a certain level of actual hardware design in power electronic circuit, one hardware project (in addition to lab experiments) is assigned for the first class. A topic in low power dc-dc converter is chosen for the hardware

October 28 - 31, 2006, San Diego, CA 
project, i.e. design of step up converter (Boost) using any commercially available switching regulator. The use of readily available switching regulator provides the following benefits: it is very practical since this type of design is usually done in industry at the system level; and the hardware design project provides students with the ability to apply their system level design skills in the dc-dc converter without having to know the details of low-level analog circuitry which is beyond the scope of the course (covered in analog courses) and may discourage students from taking the course. The week before the finals week, students then demo their hardware project which is graded based on several criteria such as how well the converter meets the design requirements set by the instructor, aesthetic and layout. In addition, the students are also required to show-case their circuit by creating a website for their circuit. Instructor will then put together all the websites into one final website which will then be announced to our electrical engineering student mailing list. This should help promote the class to the whole body of electrical engineering students. Figure 1 shows one converter circuit from the past EE 410 class hardware project.

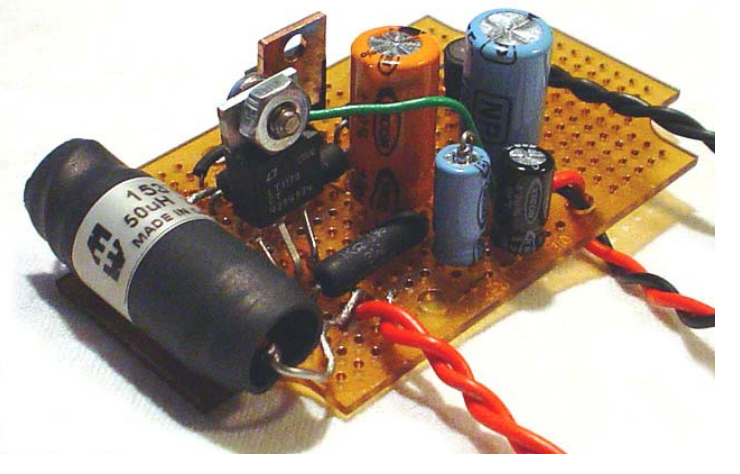

FIGURE 1

Boost converter hardware project in EE 410

In the second course EE 411, naturally more advanced topics should be covered. However, the approach here is to start adding examples in high-power (utility) applications. The main idea is now that students learned from the first course that power electronics goes beyond traditional power engineering, it is then time to teach them that power engineering is not old-fashioned. This is the course where students get to see that power engineering is not all about building power plants, putting conductors on huge power towers, transformer and rotating machines. Rather, it is a branch of electrical engineering that covers a broad spectrum of power and it is the area that is continually challenged and in need of state of the art technology for its improvement. An example such as the issue in efficiency of Intel's $90 \mathrm{~W}$ Xeon MV processor [5] provides a very good classroom discussion.

The following list topics covered in the newly redesigned second course EE 411:

- $\quad$ Switching Losses

- Snubber Circuits

- Soft-switching non-isolated topologies

1-4244-0257-3/06/\$20.00 @ 2006 IEEE
- Soft-switching isolated topologies

- DC Transmission

- Flexible AC Transmission Controllers

- Power Quality

In the second course, a hardware project is also assigned in addition to the hardware experiments. Here, students are challenged to perform a lower level system design of another dc-dc converter. They are required to build a step-down (buck) converter using an individual controller rather than switching regulator. This project is practical in a sense that the design is commonly done in industry where design specifications can not be met by readily available dc-dc converter modules, and hence the converter has to be designed from "scratch". As in the first course, students demo their project and show-case it on their website which is then put together and announced to the rest of electrical engineering students. Figure 2 shows an example of a converter circuit that students did in this class.

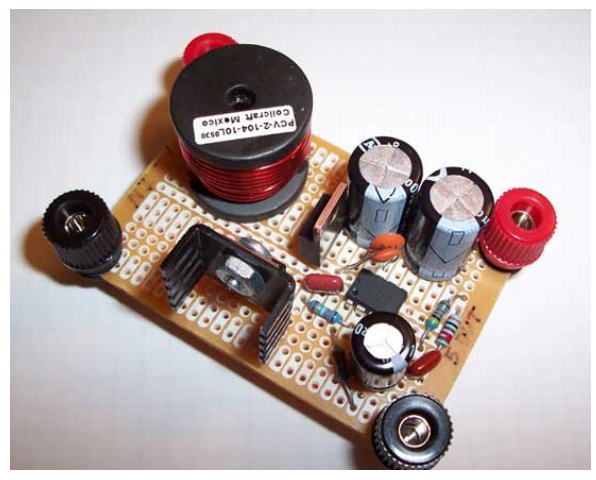

FIGURE 2

Buck converter hardware project in EE 411

Another new approach implemented in both EE 410 and EE 411 is the strong element in Computer Simulation. In both classes, students were taught to use computer simulation tool using industry standard circuit analysis software for their homework assignments and laboratory experiments. This provides another angle of attraction to electrical engineering students, since many of them do want to learn this tool in more detail than what they have been exposed to in the earlier courses.

The last course EE 527, as previously mentioned, had not been offered for years prior to 2002 due to extremely low enrollment. The approach taken here is to make the course content flexible. The course used to cover topics related to medium and high power such as three-phase inverters. After the redesign, the course will now cover any advanced topics in power electronics. With the author, the course content focuses on advanced topics in switched-mode power supply design. The topic was chosen because of the increased demand from industry for people with background in power supply design. In addition, at this point, power students normally take the more conventional power related course as their last technical elective. So, rather than competing with other power courses,

\section{$36^{\text {th }}$ ASEE/IEEE Frontiers in Education Conference}

October 28 - 31, 2006, San Diego, CA 
this course is now made open to all electrical engineering students. Here is the list of topics covered in EE 527:

- Input Stage of Power Supply

- Power Stage of Power Supply

- Output Stage of Power Supply

- Control Stage of Power Supply

In this last course a hardware project involving the design of isolated topology on a printed circuit board is assigned. Just like the previous two courses, students will have to demo and show-case their project. Figure 3 shows one circuit designed by a student in this class.

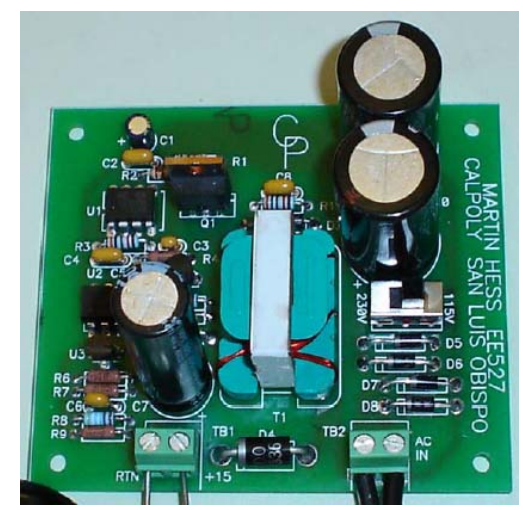

FIGURE 3

Flyback converter hardware project in EE 527

\section{REDESIGNING THE LAB EXPERIMENTS}

The first two courses (EE 410 and EE 411) have lab component embedded in them. Students are required to attend a 3-hour lab session every week for each class. When developing the new lab experiments, the following approaches were implemented:

- Each lab experiment should have three components: prelab design calculation, computer simulation to verify the prelab design, and hardware to prove the design

- Each lab experiment should focus on overall system concept, characteristics, properties and functionalities, rather than focusing on low level analog circuitry design

- Each lab experiment should required a group report

The first item is essential in teaching students practical design experience where an engineer typically start with their own design calculations and then simulate it before the hardware is actually built. The second item is important so that focus will remain on the system level hence consistent with the lecture portion of the course. At this level, students have already gone through quite a few analog circuitry labs, so system level experiments would be most appropriate and enticing for students.

Currently, in the first course EE 410, 5 experiments have been designed and one more is underway:
- $\mathrm{AC}$ to fixed de rectifier

- $\mathrm{AC}$ to adjustable de rectifier

- $\mathrm{AC}$ to $\mathrm{AC}$ controller for light-dimmer

- $\quad \mathrm{DC}$ to DC converter

- DC to single-phase AC converter (near completion)

- DC motor drive

In the second course EE 411, three lab experiments have been completed and three more are underway:

- Voltage doubler rectifier

- Synchronous rectifier (in progress)

- ZVS-Phase Shifted Bridge converter (in progress)

- Active Power Factor correction

- Static VAR compensator (near completion)

- AC motor drive

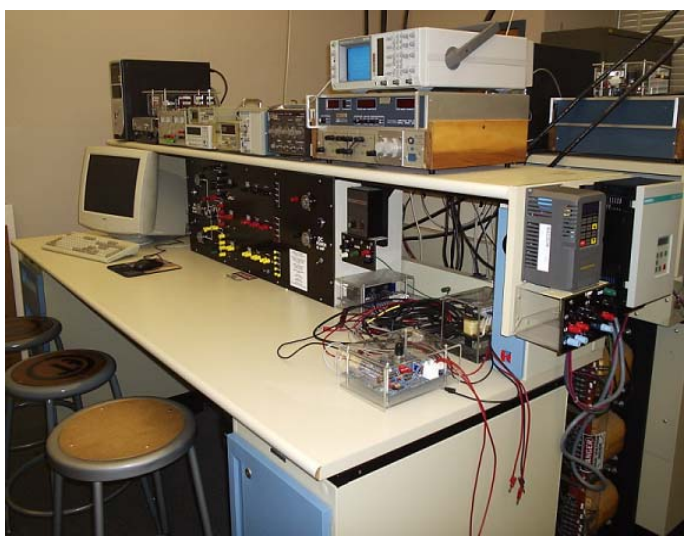

FIGURE 4

Lab bench in Power Electronics Lab

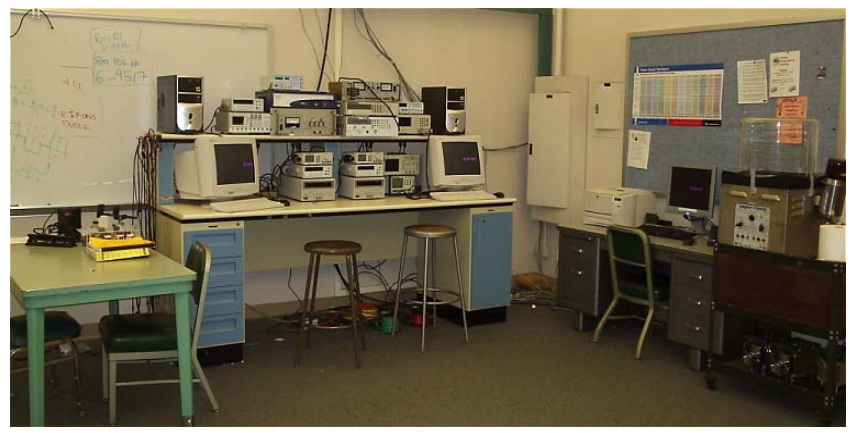

FIGURE 5

Power Electronics Senior Project Lab bench

The author strongly believes that the lab elements of power courses play a very important role in drawing electrical engineering students into studying power. It is therefore very crucial to design lab experiments that implement state of the art technology and to incorporate modern lab equipment. Currently, the power electronics lab at Cal Poly has a total of six lab benches as shown in Figure 4. In addition, since joining Cal Poly, the author has successfully obtained funding and support from local industries to build a power electronic senior

October 28 - 31, 2006, San Diego, CA 
Session M2D

project bench which is presented every year to students during the open-house week to further promote the courses. Figure 5 shows the senior project power electronics bench.

\section{IMPACT ON ENROLLMENT}

The impacts of the newly designed power electronic courses to student enrollments in the courses for the past several years are illustrated in Figures 6 to 8. The data were obtained while the enrollment in the Electrical Engineering department has remained about the same; around 700 students since 1999. Meanwhile, the number of technical elective courses offered to EE students has increased as well. Several observations were summarized as follows. All three courses have successfully attracted more students and hence increasing their enrollments. With the exception of EE 527, the majority of students taking the courses are power students. Only the first course EE 410 has attracted non-EE major, although not to a significant degree. It was observed that the non-EE students taking EE 410 were usually from the Mechatronics major. Implicitly, these figures also show that these newly redesigned courses have helped in rejuvenating the power program.

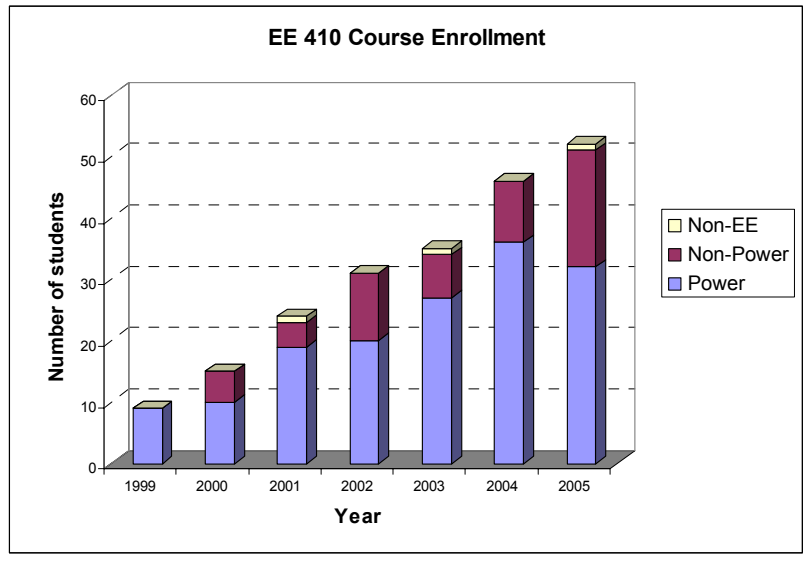

FIGURE 6

Course enrollment in EE 410

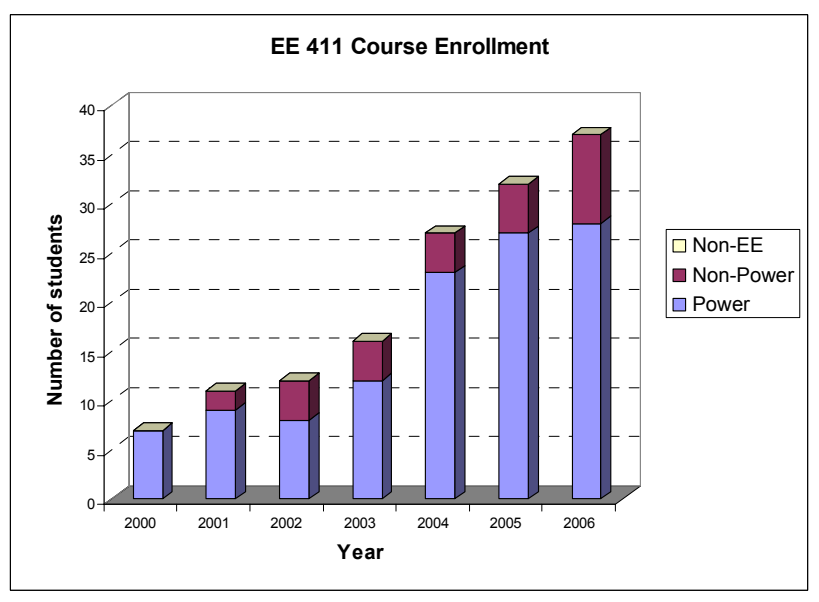

FIGURE 7

Course enrollment in EE 411
These figures also show that more than half of the students that took EE 411 continue to take the second course EE 411. However, only a small portion actually went on to take the third class EE 527. This is due to the fact that EE 527 is a graduate level course which is also open for undergraduate students. Just as in other graduate courses, the content is much more focused or narrower than those covered in EE 410 and EE 411. Hence, only the "hard-core power electronic" students will take this course. This should further explain why the number of power students in EE 527 is generally less than that of the non-power students. From a bigger perspective, however, EE 527 is a success since its enrollment has been higher than 10, more than doubled that prior to 2002. In fact, EE 527 is now considered a graduate course with enrollment higher than average enrollment of graduate level courses in the EE department at Cal Poly.

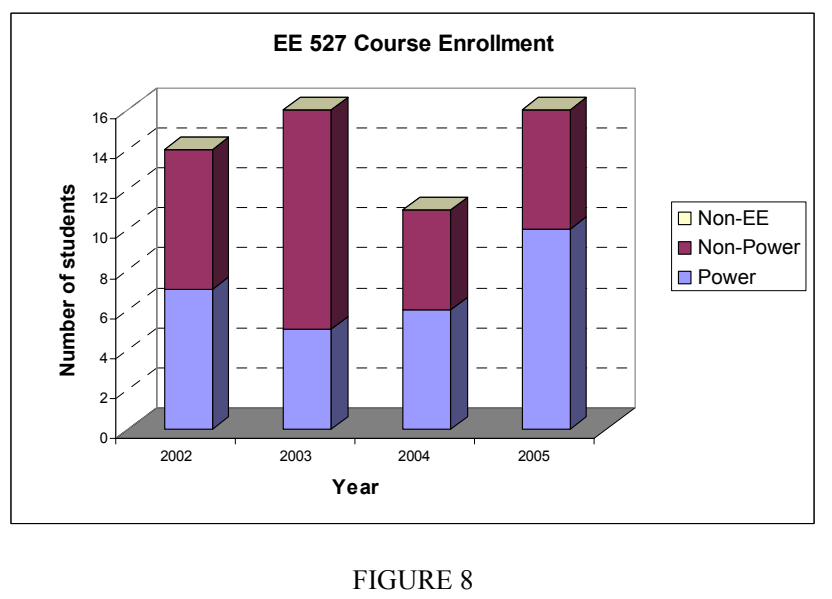

Course enrollment in EE 527

\section{CONCLUSIONS AND FURTHER WORK}

In this paper, new approaches to three courses in power electronics have been described. The results over the past six years have shown that the newly redesigned courses have worked well. The courses are now very popular technical elective courses with course enrollments in the first two courses typically above the enrollment in other technical elective courses in the EE department.

With the growth in enrollment comes the issue with accommodating these students in the labs. Currently, the power electronics lab has a total of six lab benches. This means the courses will now have to be offered with multiple lab sections. Help from a well-qualified graduate student should also be sought to aid during lab sessions.

There is still a lot of room for improvement for these three courses. The author has put together the lecture notes for each class since currently there is no single text book that covers all topics in these three courses. By far, these lecture notes are made available to students through the author's website.

Lab experiments pose another problem. It takes at least one year to design and develop a new lab experiment. As

\section{1-4244-0257-3/06/\$20.00 @ 2006 IEEE}

\section{$36^{\text {th }}$ ASEE/IEEE Frontiers in Education Conference}

October 28 - 31, 2006, San Diego, CA 
discussed in this paper, there are still few more labs need to be completed for the lab portions of these courses. One common problem with designing new power electronic lab experiment is the component and equipment needed for the experiment. The author has been fortunate that due to these newly redesigned courses, a number of industries are willing to help and support the development of new lab experiments. Figure 9 shows the new Active Power Factor experiment used in EE 411 that was made possible through industry supports (Texas Instruments and Rantec). Currently, a new lab experiment in Static VAR compensator will be developed with the help from another power electronics company.

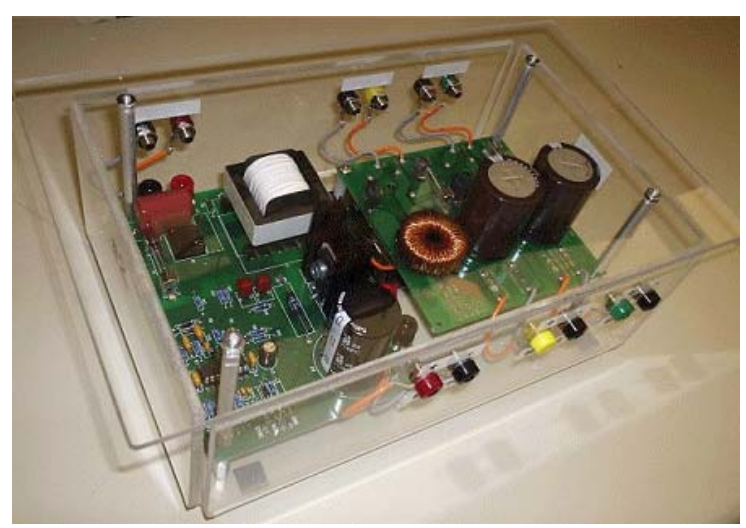

FIGURE 9

Active Power Factor Correction Module

\section{REFERENCES}

[1] Mervis, J., "Undergraduate Education: Undergraduate Data Show a Shift, Not a Decline, in Interest”, Science Magazine, Vol. 294. no. 5551, p. 2467.

[2] "Utility Industry Unites to Address Need for Skilled Workforce", Utility Business Education Coalition, Vol. 4, No 1, Spring 2005, p. 1.

[3] Mohan, N., Undeland, T., and Robbins, W., Power Electronics: Converters, Applications, and Design, $3^{\text {rd }}$ Edition, John Wiley \& Sons, 2003.

[4] Joos, G., "Training Future Power Engineers", IEEE Power and Energy Magazine, Vol. 3, No. 1, Jan/Feb 2005, p. 39.

[5] Intel Corp., "Intel Pushes Xeon”, byteandswitch.com, Sept. 26, 2005. 